\title{
Urinary trypsin inhibitor protects against liver injury and coagulation pathway dysregulation induced by lipopolysaccharide/D-galactosamine in mice
}

\author{
Hirohisa Takano ${ }^{1,2,5}$, Ken-ichiro Inoue ${ }^{1,5}$, Akinori Shimada ${ }^{3}$, Hiroyuki Sato ${ }^{4}$, Rie Yanagisawa ${ }^{1}$ and \\ Toshikazu Yoshikawa ${ }^{2}$
}

Urinary trypsin inhibitor (UTI), a serine protease inhibitor, has been widely used for patients with inflammatory disorders including disseminated intravascular coagulation, shock, and pancreatitis in Japan. Our recent studies using UTI-null $(-/-)$ mice have shown that UTI protects against systemic inflammatory responses and acute lung injury. However, the role of UTI in liver injury has not been elucidated. This study determined the contribution of UTI to liver injury and coagulatory disturbance induced by lipopolysaccharide and D-galactosamine (LPS/D-GaIN) using UTI (-/-) and wild-type (WT) mice. LPS/D-GalN treatment caused severe liver injury characterized by neutrophilic inflammation, hemorrhagic change, necrosis, and apoptosis, which was more prominent in UTI (-/-) than in WT mice. In both genotypes of mice, LPS/D-GalN challenge caused elevations of aspartate amino-transferase and alanine amino-transferase, prolongation of the prothrombin and activated partial thromboplastin time, and decreases in fibrinogen and platelet counts, as compared with vehicle challenge. These changes, however, were significantly greater in UTI (-/-) than in WT mice. Circulatory levels of tumor necrosis factor (TNF)- $\alpha(P<0.05)$ and interferon (IFN)- $\gamma$ were also greater in UTI $(-/-)$ than in WT mice after LPS/D-GalN challenge. These results suggest that UTI protects against severe liver injury and subsequent coagulatory disturbance induced by LPS/D-GalN, which was mediated, at least partly, through the suppression of TNF- $\alpha$ production along with its antiprotease activity.

Laboratory Investigation (2009) 89, 833-839; doi:10.1038/labinvest.2009.35; published online 27 April 2009

KEYWORDS: urinary trypsin inhibitor; lipopolysaccharide; D-Galactosamine; liver injury; disseminated intravascular coagulation

Urinary trypsin inhibitor (UTI) is a multivalent Kunitz-type serine protease inhibitor that is found in human urine and blood. ${ }^{1}$ UTI has been widely used as a drug for patients with disseminated intravascular coagulation (DIC), shock, and pancreatitis, especially in Japan. ${ }^{1}$ UTI mainly inhibits inflammatory proteases including trypsin, $\alpha$-chymotrypsin, plasmin, cathepsin G, and leukocyte elastase as well as proteases in the coagulation cascade. Furthermore, UTI reportedly exhibits anti-inflammatory properties aside from its blocking of the protease pathway in vitro ${ }^{2-4}$ and in vivo. ${ }^{5-7}$
In fact, we demonstrated earlier that UTI can protect against systemic inflammatory responses ${ }^{8}$ and acute lung injury ${ }^{9}$ induced by lipopolysaccharide (LPS) using UTI-null (-/-) mice. However, the role of UTI in the other inflammatory conditions and/or organ injury has not been elucidated using gene-knockout mice.

Fulminant hepatic failure is associated with a mortality rate of $82-97 \%$, if liver transplantation is not available. ${ }^{10} \mathrm{~A}$ variety of toxins accumulating in the body as a result of liver injury and cytokines released directly from the necrotic liver

\footnotetext{
'Division of Environmental Health Sciences, National Institute for Environmental Studies, Tsukuba, Japan; ${ }^{2}$ Department of Gastroenterology, Graduate School of Medical Science, Kyoto Prefectural University of Medicine, Kyoto, Japan; ${ }^{3}$ Department of Veterinary Pathology, Faculty of Agriculture, Tottori University, Tottori, Japan and ${ }^{4}$ Research Center, Mochida Pharmaceutical Company Ltd., Shizuoka, Japan

Correspondence: Dr K-i Inoue, MD, PhD, Division of Environmental Health Sciences, National Institute for Environmental Studies, 16-2 Onogawa, Tsukuba 305-8506, Japan.

E-mail: inoue.kenichirou@nies.go.jp

${ }^{5}$ These authors contributed equally to this work.
}

Received 29 August 2008; revised 10 November 2008; accepted 21 November 2008 
into the circulation are considered to be responsible for the deterioration of the condition. ${ }^{11}$ It is also reported that activated leukocytes and their proteases can contribute to the pathogenesis of liver injury. ${ }^{12,13}$ Furthermore, severe liver injury develops both impaired productive and consumptive coagulatory disturbance resulting in DIC and fatal outcomes. $^{14,15}$

In this study, we explored the role of UTI in severe liver injury induced by the intraperitoneal injection of LPS/Dgalactosamine (D-GalN) using UTI $(-/-)$ mice and the corresponding wild-type (WT) mice. We also determined the effects of UTI on coagulatory disturbance during lethal liver injury. Furthermore, we examined whether liver injury with coagulatory disturbance is concomitant with altered profiles of proinflammatory cytokines. Finally, we elucidated the effects of UTI on apoptosis and oxidative stress related to LPS/D-GalN.

\section{MATERIALS AND METHODS \\ Mice}

The generation of mice deficient in the UTI gene and normal control littermates (C57BL/6) was described earlier. ${ }^{16}$ These mice were bred and maintained under a 12-h light-dark cycle in our Level B pathogen-free facility. Male mice of both genotypes were used at $10-12$ weeks of age and $27-31 \mathrm{~g}$ in weight.

\section{LPS/D-GalN Challenge}

Both UTI $(-/-)$ and WT mice were injected intraperitoneally (i.p.) with phosphate-buffered saline (PBS) at $\mathrm{pH} 7.4$ (Invitrogen Co., Carlsbad, CA) or $3 \mu \mathrm{g} / \mathrm{kg}$ of LPS (Escherichia coli B55: 05, Difco Lab, Detroit, MI) and $800 \mathrm{mg} / \mathrm{kg}$ of D-GalN dissolved in PBS. The studies were carried out in accordance with the Guide for the Care and Use of Laboratory Animals as adopted and promulgated by the National Institute of Health. All animal studies were approved by the Institutional Review Board.

\section{Histological Examination}

After exsanguinations, the livers were fixed with $10 \%$ neutral phosphate-buffered formalin ( $\mathrm{pH}$ 7.4). All specimens were embedded in paraffin. Sections of $4 \mu \mathrm{m}$ thick were routinely processed with hematoxylin and eosin stain $(n=4$ in each group). Neutrophil infiltration was assessed by averaging the number of neutrophils enumerated in 30 randomly selected high-power fields $(\times 400)$ in each slide. Histological sections were evaluated in a blind fashion.

\section{Immunohistochemistry}

The detection of apoptosis in the liver was examined by immunohistochemical analysis ( $n=4$ in each group) using an anti-single-stranded DNA (ss-DNA) polyclonal antibody (Dako Japan, Kyoto, Japan) according to a previous study, ${ }^{17}$ because ss-DNA is accepted to be another maker of DNA fragmentation. ${ }^{18,19}$ Deparaffinized slides were blocked with
$10 \%$ goat serum for $1 \mathrm{~h}$. After blocking, anti-ss-DNA antibody $(0.5 \mu \mathrm{g} / \mathrm{ml})$ was incubated with the sections for $1 \mathrm{~h}$ at room temperature, followed by the incubation of a biotinylated secondary antibody and streptavidin-peroxidase conjugate. Thereafter, the slides were incubated with 3-amino, 9-ethyl-carbazole chromogen, and counterstained with hematoxylin in an AutoProbe III kit (Biomeda, Foster City, CA).

\section{Analysis for Coagulation and Fibrinolysis Parameters, Peripheral Blood Cell Counts, and Liver Transaminases} Blood samples were collected from each mouse $(n=4-6$ in each group) into $3.8 \%$ sodium citrate at a ratio of $10: 1$ and centrifuged at $3000 \mathrm{~g}$ for $10 \mathrm{~min}$, as conducted earlier. ${ }^{8}$ The prothrombin time (PT) was evaluated by incubating $50 \mu \mathrm{l}$ of plasma for $5 \mathrm{~min}$ at $37^{\circ} \mathrm{C}$ and then adding $100 \mu \mathrm{l}$ of Neoplastin Plus (DIAGNOSTICA STAGO, Roche, Tokyo, Japan) and $30 \mathrm{mmol} / \mathrm{l}$ of $\mathrm{CaCl}_{2}$. The activated partial thromboplastin time (APTT) was measured by mixing $50 \mu \mathrm{l}$ of STA APTT LT (DIAGNOSTICA STAGO, Roche) and $50 \mu \mathrm{l}$ of murine plasma followed by incubation for $4 \mathrm{~min}$ at $37^{\circ} \mathrm{C}$ and decalcification with $50 \mu \mathrm{l}$ of $25 \mathrm{mmol} / \mathrm{l}$ of $\mathrm{CaCl}_{2}$. Murine clottable plasma fibrinogen was determined using a commercial kit (DIAGNOSTICA STARGO, Roche) and the values were compared with a human plasma fibrinogen standard (DIAGNOSTICA STARGO, Roche). All assays were measured using STA Compact (DIAGNOSTICA STAGO, Roche), as described earlier. ${ }^{8,20,21}$

In a separate series of experiments, blood samples were collected and peripheral blood cell counts, aspartate aminotransferase (AST), and alanine amino-transferase (ALT) were measured ( $n=10$ in each group).

\section{Assays for Circulatory Cytokines}

Blood samples were collected from each mouse $(n=9-10$ in each group) and centrifuged at $2500 \mathrm{~g}$ for $10 \mathrm{~min}$. ELISA for tumor necrosis factor (TNF) $-\alpha$ (R \& D Systems, Minneapolis, $\mathrm{MN}$ ) and interferon (IFN)- $\gamma$ (Endogen, Cambridge, MA) in the serum were conducted using matching antibody pairs according to the manufacturer's instructions. The secondary antibodies were conjugated with horseradish peroxidase. Readings at $550 \mathrm{~nm}$ subtracted from those at $450 \mathrm{~nm}$ were converted to $\mathrm{pg} / \mathrm{ml}$ using values obtained from standard curves generated with varying concentrations of recombinant TNF- $\alpha$ and IFN- $\gamma$ with detection limits of 3.8 and $2 \mathrm{pg} / \mathrm{ml}$, respectively.

\section{Statistical Analysis}

Data are reported as the mean \pm s.e.m. using Stat view version 4.0 (Abacus Concepts Inc., Berkeley, CA). Differences between groups in PT, APTT, fibrinogen, and neutrophil number in the liver sections were analyzed by the KruskalWallis test followed by Mann-Whitney $U$-test using Stat view. Differences in other data were examined for significance 
using one-way ANOVA with post hoc analysis (Fisher's PLSD test). Significance was assigned to $P$-values smaller than 0.05 .

\section{RESULTS}

\section{Role of UTI in Liver Injury Caused by LPS/D-GaIN Challenge}

We evaluated the histopathological changes in livers obtained from both genotypes of mice $5 \mathrm{~h}$ after LPS/D-GalN challenge (Figure 1). Histopathological examination revealed severe liver injury characterized by neutrophilic infiltration, hemorrhagic change, apoptosis, and necrosis in UTI $(-/-)$ mice challenged with LPS/D-GalN, whereas the liver injury was less prominent in WT mice. Vehicle treatment caused no histopathological changes in both genotypes of mice. We performed morphometric analysis to quantitate the number of neutrophils that had infiltrated into the liver. As compared with vehicle treatment (mean \pm s.e.m.: $0.6 \pm 0.2$ for WT, $0.5 \pm 0.2$ for UTI $[-/-]$ ), LPS/D-GalN treatment significantly increased the number of neutrophils from both genotypes of mice $(21.2 \pm 8.6$ for WT, $32.9 \pm 9.8$ for UTI $[-/-] ; P<0.01)$. In the presence of LPS/D-GalN, the number was greater in UTI $(-/-)$ mice than in WT mice $(P=0.08)$.

\section{Role of UTI in the Induction of Apoptosis in the Liver Induced by LPS/D-GalN Challenge}

To elucidate the role of UTI in liver cell apoptosis induced by LPS/D-GalN, the induction of apoptosis was estimated $5 \mathrm{~h}$ after intraperitoneal administration (Figure 2). LPS/D-GalN challenge induced apoptosis in the livers from both genotypes of mice. However, the intensity was apparently stronger in UTI $(-/-)$ than in WT mice.

\section{Effects of UTI Deficiency on the Changes in Liver Transaminases, Coagulatory and Fibrinolytic Parameters, and Peripheral Blood Cell Counts after LPS/ D-GaIN Challenge}

We next evaluated the levels of liver transaminases, coagulatory and fibrinolytic parameters, and peripheral blood cell counts $5 \mathrm{~h}$ after intraperitoneal challenge with LPS/D-GalN or vehicle (Figure 3). LPS/D-GalN treatment showed elevations of liver transaminases as compared with vehicle treatment, especially in UTI $(-/-)$ mice $(P<0.01$ for UTI $[-/-])$. In the presence of LPS/D-GalN, the levels of AST and ALT were significantly greater in UTI $(-/-)$ than in WT mice $(P<0.01)$. LPS/D-GalN challenge significantly prolonged PT
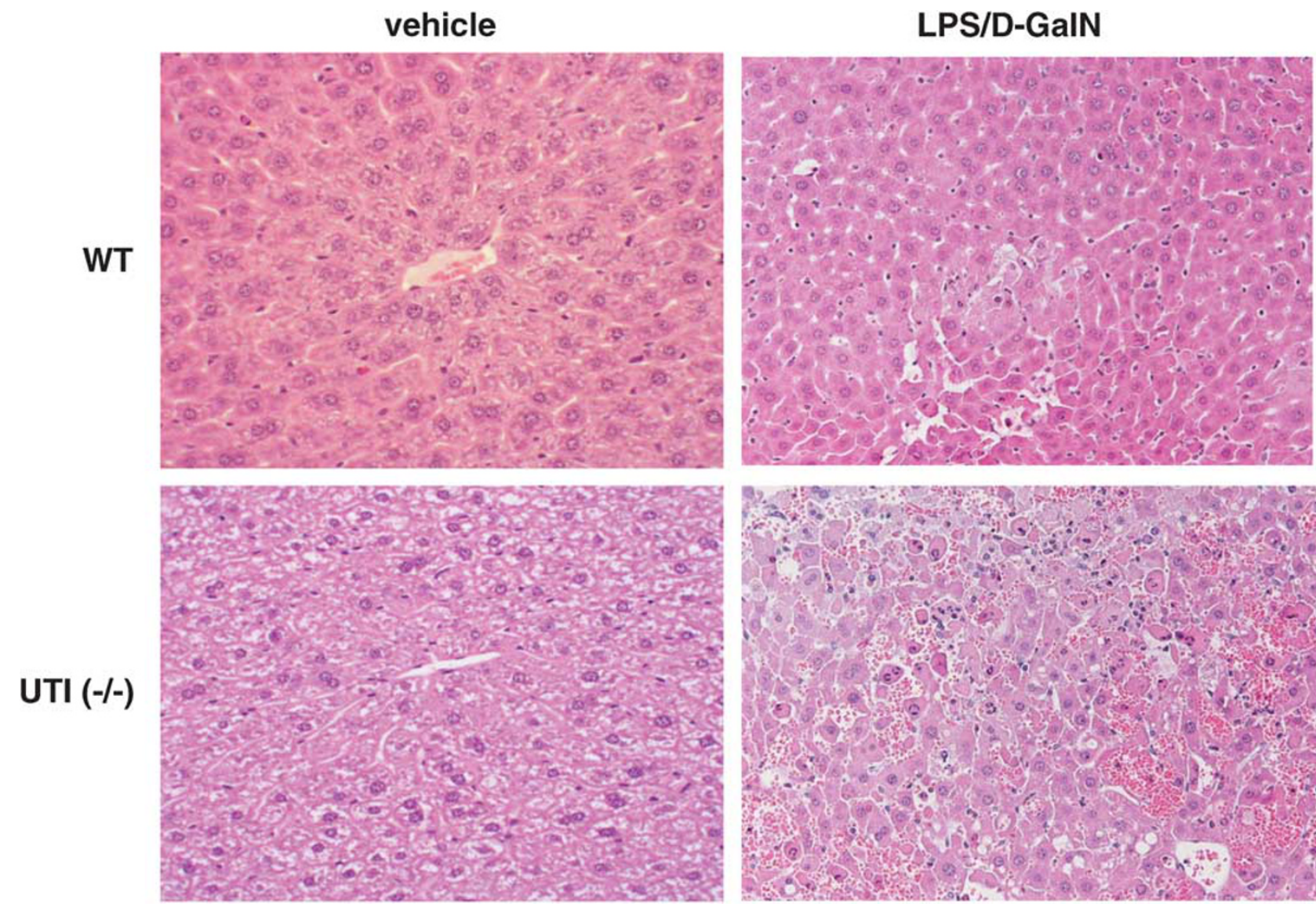

Figure 1 Histopathological findings of the liver obtained from urinary trypsin inhibitor (UTI) null (-/-) mice injected i.p. with $3 \mu \mathrm{g} / \mathrm{kg}$ body weight of lipopolysaccharide (LPS) and $800 \mathrm{mg} / \mathrm{kg}$ of D-galactosamine (D-GaIN), wild-type (WT) mice injected with LPS/D-GalN, UTI (-/-) mice injected with vehicle, and WT mice injected with vehicle ( $n=4$ in each group). Five $h$ after injection, mice were killed and examined. Original magnification $\times 200$. 

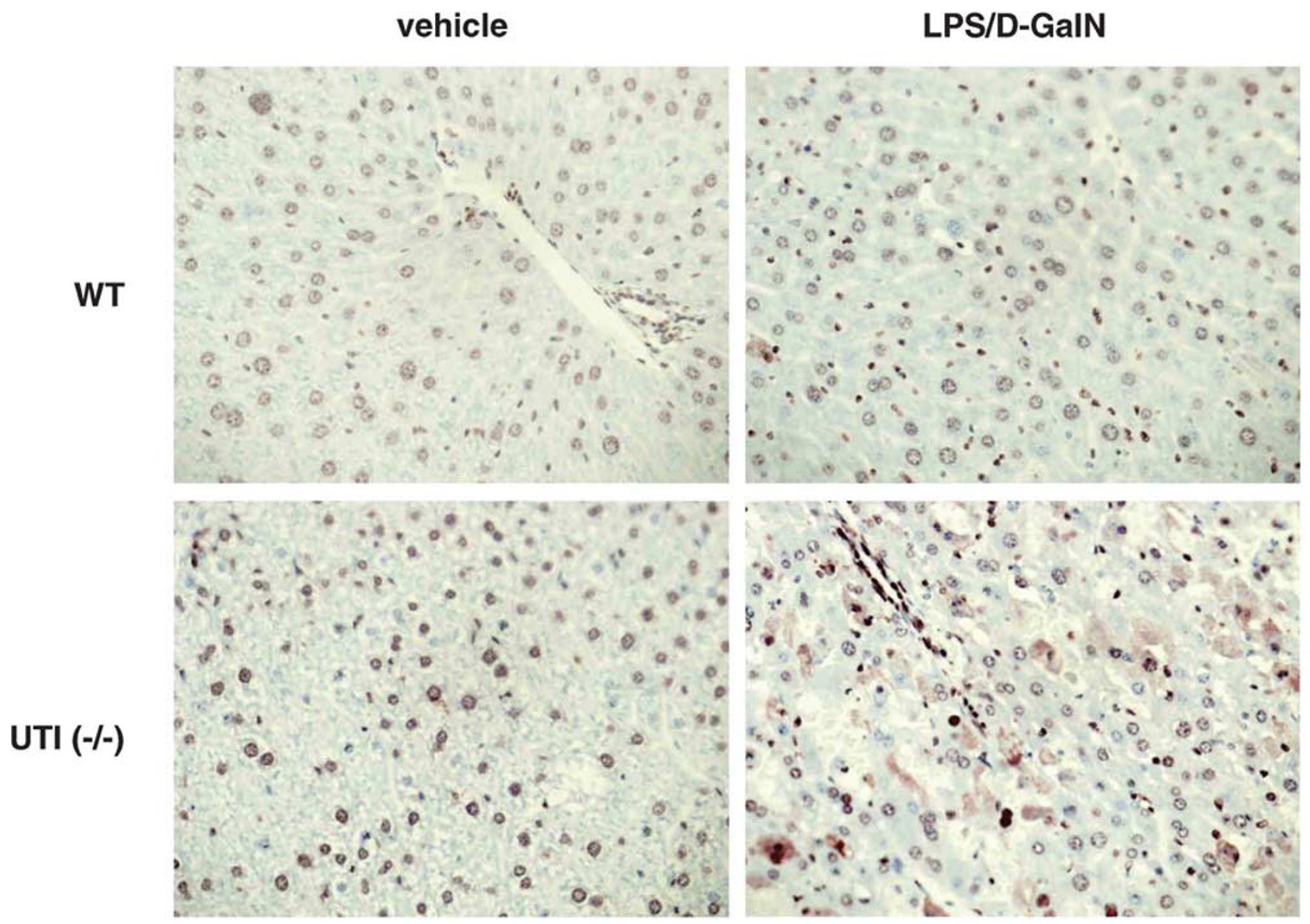

Figure 2 Immunohistochemical staining for single-stranded DNA of the liver obtained from UTI (-/-) mice injected with LPS/D-GalN, WT mice injected with LPS/D-GaIN, UTI (-/-) mice injected with vehicle, and WT mice injected with vehicle ( $n=4$ in each group). Five h after injection, mice were killed and examined. Arrows denote positive staining. Original magnification $\times 300$.

in both genotypes of mice as compared with vehicle challenge $(P<0.01)$. In the presence of LPS/D-GalN, PT was significantly longer in UTI $(-/-)$ than in WT mice $(P<0.01)$. APTT was also significantly longer in LPS/D-GalN-treated UTI $(-/-)$ than in vehicle-treated UTI $(-/-)(P<0.01)$ or LPS/D-GalN-treated WT mice $(P<0.05)$. Levels of fibrinogen and counts of platelets were significantly lower in LPS/DGalN-challenged than in vehicle-challenged mice in the same genotypes $(P<0.05$ for WT, $P<0.01$ for UTI $[-/-])$. In the presence of LPS/D-GalN, they were significantly lower in UTI $(-/-)$ than in WT mice $(P<0.01)$. As compared with vehicle challenge, LPS/D-GalN challenge significantly decreased white blood cell (WBC) counts only in UTI $(-/-)$ mice $(P<0.05)$, not in WT mice.

\section{Role of UTI in Circulatory Levels of TNF- $\alpha$ and IFN- $\gamma$ after LPS/D-GaIN Challenge}

To determine the role of UTI in the systemic inflammatory response related to LPS/D-GalN, levels of serum TNF- $\alpha$ and IFN- $\gamma$ proteins were estimated $5 \mathrm{~h}$ after intraperitoneal administration (Figure 4). The level of TNF- $\alpha$ was greater in
LPS/D-GalN-treated than in vehicle-treated mice $(P<0.05$ for UTI $[-/-])$. In the presence of LPS/D-GalN, the level was significantly greater in UTI $(-/-)$ than in WT mice $(P<0.05)$. The level of IFN- $\gamma$ was also greater in LPS/DGalN-treated than in vehicle-treated mice $(P<0.05$ for UTI $[-/-])$. In the presence of LPS/D-GalN, the level was greater in UTI $(-/-)$ than in WT mice, however, it did not reach significance.

\section{DISCUSSION}

This study demonstrated that LPS/D-GalN challenge induces severe liver injury characterized by hemorrhagic change, necrosis, apoptosis, and neutrophilic infiltration, which is far more prominent in UTI $(-/-)$ than in WT mice. As compared with WT mice, UTI $(-/-)$ mice show significant elevations of liver transaminases such as AST and ALT, significant prolongations of PT and APTT, and significant decreases in fibrinogen, WBC, and platelet counts after LPS/ D-GalN challenge. Circulatory levels of IFN- $\gamma$ and TNF- $\alpha$ are greater in UTI $(-/-)$ than in WT mice after LPS/D-GalN challenge. 

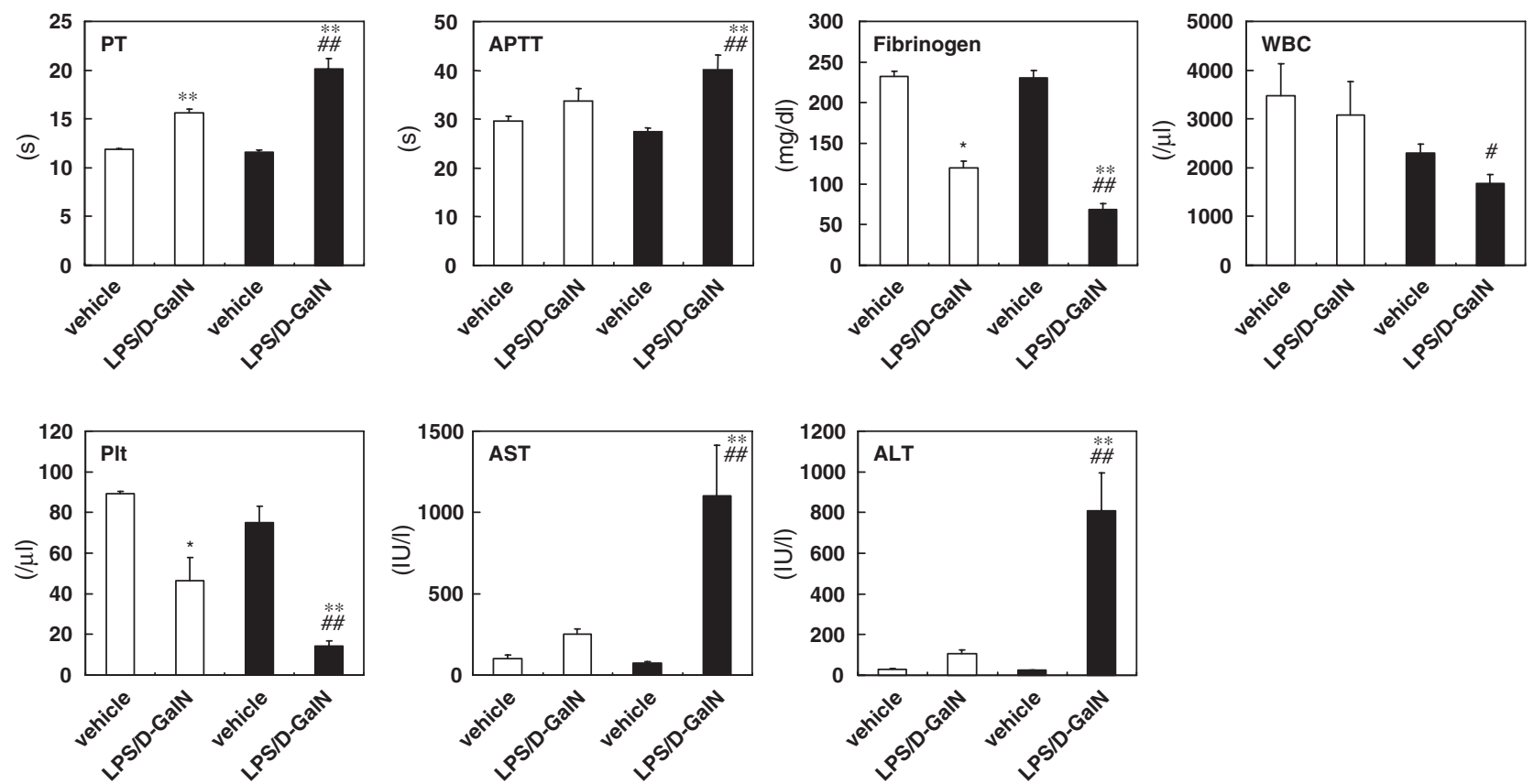

Figure 3 Changes in coagulatory parameters, peripheral blood cell counts, and liver transaminases after LPS/D-GalN challenge. Blood samples were collected $5 \mathrm{~h}$ after i.p. injection of vehicle or LPS/D-GalN. Coagulatory parameters ( $n=4-6$ in each group), white blood cell (WBC) and platelets counts, and the levels of liver transaminases ( $n=10$ in each group) in the sera were measured. ${ }^{\star} P<0.05$ vs vehicle-treated mice; ${ }^{* *} P<0.01$ vs vehicle-treated mice;

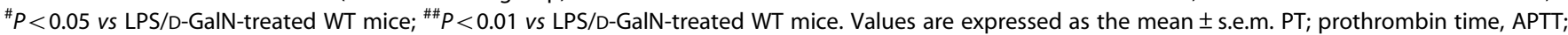
activated partial thromboplastin time, AST; aspartate amino-transferase, ALT; alanine amino-transferase.
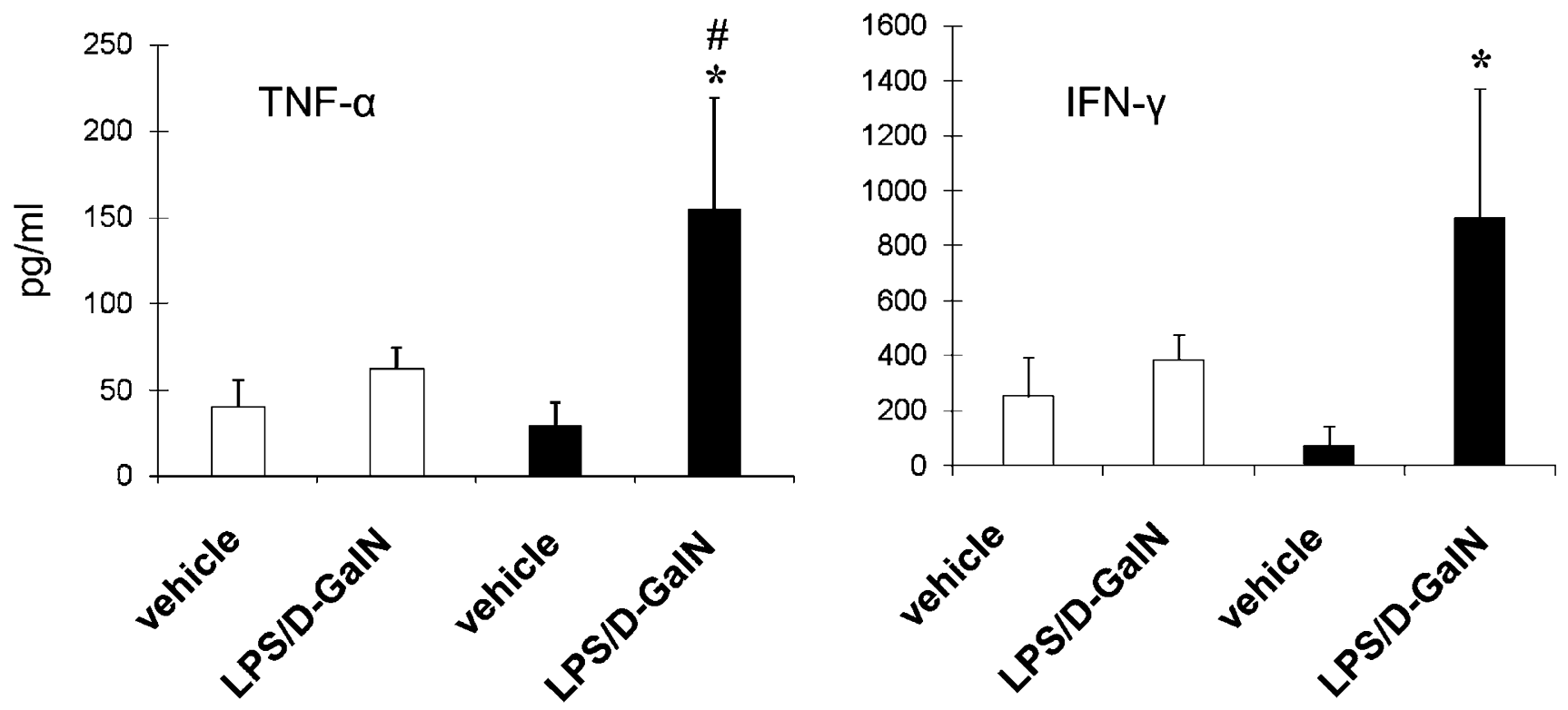

Figure 4 Serum levels of cytokines after LPS/D-GalN challenge. Sera of both WT (open symbols) and UTI (-/-: filled symbols) mice were harvested $5 \mathrm{~h}$ after i.p. injection of vehicle or LPS/D-GaIN ( $n=9-10$ in each group). Tumor necrosis factor- $\alpha$ and interferon- $\gamma$ levels were measured by ELISA. ${ }^{\star} P<0.05$ vs vehicletreated mice, ${ }^{\#} P<0.05$ vs LPS/D-GalN-treated WT mice. Values are the mean \pm s.e.m. in each group.

UTI is a multivalent Kunitz-type serine protease inhibitor. Although the therapeutic effects of UTI on circulatory shock and pancreatitis have been recognized, especially in Japan, ${ }^{1}$ detailed explanations of the mechanisms remain unsatisfactory. Tani et $a l^{6}$ have reported that UTI protects against septic shock induced by Gram-negative bacteria 
in vivo, by estimating clinical signs such as the cardiac index, blood pressure, lactic acid, blood glucose, and blood baseline values. Another group has reported that UTI improves hemorrhagic shock through its protective effect on myocardial mitochondrial functions. ${ }^{7}$ Previously, we showed the protective role of UTI in two models related to inflammation using gene-knockout mice. In our first report, UTI protected against systemic inflammation including hyperfibrinogenemia induced by the intraperitoneal administration of LPS. ${ }^{8}$ Also, the aggravated systemic inflammation in UTI (-/-) mice was associated with the more prominent inflammatory organ (lung, liver, and kidney) damage and cytokine expressions in the organs. Secondly, UTI $(-/-)$ mice revealed aggravated lung injury induced by the intratracheal administration of LPS. ${ }^{9}$ The deteriorated lung injury in UTI $(-/-)$ mice was concomitant with the enhanced lung expression of proinflammatory cytokines such as IL- $1 \beta$, chemokines such as macrophage inflammatory protein (MIP)- $1 \alpha$, macrophage chemoattractant protein (MCP)-1, keratinocyte chemoattractant $(\mathrm{KC})$, and intercellular adhesion molecule- 1 .

On the other hand, activated neutrophils release various kinds of mediators, including proteases. ${ }^{22}$ Protease-antiprotease imbalance has been reportedly involved in a variety of inflammatory diseases. ${ }^{23,24}$ Harbrecht et al ${ }^{13}$ have demonstrated that liver cell damage by activated neutrophils is mediated by proteases in vitro. In addition, Sauer et al ${ }^{12}$ have reported the importance of proteases in neutrophil-mediated liver injury in vivo. Thus, this study has focused on the effects of UTI on inflammatory liver injury.

GalN is metabolized by enzymes participating in the galactose pathway. As this pathway consumes uridine nucleotide, GalN challenge leads to a rapid depletion of these nucleotides primarily in the liver, and to a decrease in RNA synthesis. Injection of a large dose of GalN into animals induces liver cell necrosis with apoptosis similar to that in human viral hepatitis. ${ }^{25}$ An earlier study in patients with acute and fulminant hepatitis has indicated the close relationship between plasma and urine levels of UTI and the severity of liver damage and corresponding coagulatory and fibrinolytic dysfunction, ${ }^{26}$ implicating UTI as a contributor to this pathological condition. In this study, liver injury induced by LPS/D-GalN was far more prominent in UTI $(-/-)$ than in WT mice. Also, in the presence of LPS/D-GalN, the levels of liver-derived transaminases such as AST and ALT were much greater in UTI $(-/-)$ than in WT mice. These results suggest that UTI should be protective against inflammatory liver injury.

Hepatic failure is often associated with coagulatory and fibrinolytic disturbance. It is, at least partly, a result of exhausted coagulatory factors synthesized by the liver. In particular, liver injury reportedly leads to the tissue factorinitiated consumption of Factor II, V, VII, and X. ${ }^{15}$ In this study, UTI $(-/-)$ mice showed a significant prolongation of PT and APTT, and significant decreases in fibrinogen and platelet counts as compared with WT mice in the presence of LPS/D-GalN. These results suggest that UTI can protect against coagulatory disturbance in fulminant hepatic failure that can result in DIC. In contrast, LPS/D-GalN-treated mice showed a septic state including a high circulatory level of proinflammatory cytokines such as TNF- $\alpha$ (Figure 3 ), IL-1 $\beta$ (data not shown), and IL-6 (data not shown), which are implicated in the development of sepsis. ${ }^{27}$ Thus, it cannot be excluded that sepsis is considerably related to the pathogenesis of DIC in this study. In other words, it could be proposed that both pathologies are attributable to the fatal outcome.

Recent studies have demonstrated that protease inhibitors may have anti-inflammatory roles rather than merely suppressive effects on protease actions during inflammation. UTI inhibits IL- $8^{4}$ and TNF- $\alpha^{28}$ in vitro. Also, in our recent study, protein levels of MCP-1 in the lungs, MCP-1 and KC in the kidneys, and IL- $1 \beta$, MIP-2, MCP-1, and KC in the liver are significantly greater in UTI $(-/-)$ than in WT mice after LPS challenge. ${ }^{8}$ On the other hand, LPS is known to stimulate the production and release of a variety of cytokines. Of these, TNF- $\alpha$ is considered to be the most responsible for the lethality seen on LPS/D-GalN challenge. ${ }^{12}$ In this study, serum TNF- $\alpha$ levels were markedly higher in UTI $(-/-)$ than in WT mice in the presence of LPS/D-GalN. These results suggest that the protective effects of UTI on LPS/D-GalN-related injury should be mediated, at least partly, through the suppression of TNF- $\alpha$ production.

Liver cell apoptosis represents not only a crucial step in acute hepatic failure, but also functions as a signal for leukocyte infiltration in parenchymal cells, ${ }^{29,30}$ thereby establishing a vicious circle with the augmentation of leukocyte inflammation and cell death, although necrosis is also likely to markedly contribute to the liver injury in view of the elevation of serum liver transaminase levels and histological findings (see Leonis et $a l^{25}$ and Figures 1 and 4 in this study). Consistent with this, preventing apoptosis in hepatocytes by caspase inhibition reportedly suppresses leukocyte transmigration and leukocyte-dependent liver cell necrosis accompanied by apoptosis. ${ }^{30}$ Furthermore, as a mechanistic hint, the sequential analysis of proapoptotic and antiapoptotic gene expression in LPS/D-GalN-exposed liver has indicated that there is an early block in the transcription of antiapoptotic Bcl-2 and Bcl- $\mathrm{X}_{\mathrm{L}}$ after the LPS/D-GalN exposure, before the proapoptotic gene Bax is transcriptionally activated. $^{31}$ On the other hand, UTI reportedly reduces lymphocyte apoptosis, ${ }^{32}$ although its mechanistic pathway remains unclear. In this study, apoptotic lesions in the liver were profoundly larger and more severe in UTI $(-/-)$ than in WT mice in the presence of LPS/D-GalN. Thus, our genetic approach also suggests that UTI can protect against apoptosis in vivo, which may, at least in part, correlate with the suppressive effects on leukocyte migration.

Finally, there is one major limitation regarding this study. We did not confirm whether exogenous UTI administration abrogates the changes to clearly show that LPS/D-GalN is acting in concert with UTI, and not independently. In 
previous studies, UTI experimentally ameliorated several inflammatory models such as ischemia-reperfusion injury, ${ }^{5}$ septic shock, ${ }^{6}$ hemorrhagic shock, ${ }^{7}$ and glomerulonephritis ${ }^{33}$ in vivo. In these models, however, the animals were treated with human-derived UTI as a foreign protein. Accordingly, this critical point should be clarified using appropriate UTI derived from corresponding species in the future.

In conclusion, UTI protects against fulminant liver injury and coagulatory disturbance induced by LPS/D-GalN, possibly through the suppression of TNF- $\alpha$ production, and apoptosis, aside from its antiprotease activity. These results provide direct molecular evidence for the 'rescue' therapeutic utility of UTI against fulminant hepatic failure, particularly, that associated with DIC.

\section{ACKNOWLEDGEMENT}

We thank Naoko Ueki and Satomi Abe for their assistance throughout the work.

\section{CONFLICT OF INTEREST}

The authors declare no conflict of interest.

1. Takubo T, Kuwajima S, Tatsumi N. [Human urinary trypsin inhibitor]. Nippon Rinsho 1999;57(Suppl):593-596.

2. Zaitsu M, Hamasaki Y, Tashiro K, et al. Ulinastatin, an elastase inhibitor inhibits the increased mRNA expression of prostaglandin $\mathrm{H} 2$ synthasetype 2 in Kawasaki disease. J Infect Dis 2000;181:1101-1109.

3. Aibiki $M$, Cook JA. Ulinastatin, a human trypsin inhibitor, inhibits endotoxin-induced thromboxane B2 production in human monocytes. Crit Care Med 1997;25:430-434.

4. Nakamura $\mathrm{H}$, Abe $\mathrm{S}$, Shibata $\mathrm{Y}$, et al. Inhibition of neutrophil elastaseinduced interleukin-8 gene expression by urinary trypsin inhibitor in human bronchial epithelial cells. Int Arch Allergy Immunol 1997:112:157-162.

5. Yano T, Anraku S, Nakayama R, et al. Neuroprotective effect of urinary trypsin inhibitor against focal cerebral ischemia-reperfusion injury in rats. Anesthesiology 2003;98:465-473.

6. Tani $\mathrm{T}$, Aoki $\mathrm{H}$, Yoshioka $\mathrm{T}$, et al. Treatment of septic shock with a protease inhibitor in a canine model: a prospective, randomized, controlled trial. Crit Care Med 1993;21:925-930.

7. Masuda T, Sato K, Noda C, et al. Protective effect of urinary trypsin inhibitor on myocardial mitochondria during hemorrhagic shock and reperfusion. Crit Care Med 2003;31:1987-1992.

8. Inoue $\mathrm{KI}$, Takano $\mathrm{H}$, Shimada A, et al. Urinary trypsin inhibitor protects against systemic inflammation induced by lipopolysaccharide. Mol Pharmacol 2005;67:673-680.

9. Inoue $\mathrm{K}$, Takano $\mathrm{H}$, Yanagisawa $\mathrm{R}$, et al. Protective role of urinary trypsin inhibitor in acute lung injury induced by lipopolysaccharide. Exp Biol Med (Maywood) 2005;230:281-287.

10. Rakela J, Lange SM, Ludwig J, et al. Fulminant hepatitis: Mayo Clinic experience with 34 cases. Mayo Clin Proc 1985;60:289-292.

11. Iwai $H$, Nagaki $M, N$ aito $T$, et al. Removal of endotoxin and cytokines by plasma exchange in patients with acute hepatic failure. Crit Care Med 1998;26:873-876.

12. Sauer A, Hartung T, Aigner J, et al. Endotoxin-inducible granulocytemediated hepatocytotoxicity requires adhesion and serine protease release. J Leukoc Biol 1996;60:633-643.
13. Harbrecht BG, Billiar TR, Curran RD, et al. Hepatocyte injury by activated neutrophils in vitro is mediated by proteases. Ann Surg 1993:218:120-128.

14. Diaz-Buxo JA, Blumenthal S, Hayes D, et al. Galactosamine-induced fulminant hepatic necrosis in unanesthetized canines. Hepatology 1997;25:950-957.

15. Kerr $R$, Newsome $P$, Germain $L$, et al. Effects of acute liver injury on blood coagulation. J Thromb Haemost 2003;1:754-759.

16. Sato H, Kajikawa $S$, Kuroda $S$, et al. Impaired fertility in female mice lacking urinary trypsin inhibitor. Biochem Biophys Res Commun 2001;281:1154-1160.

17. Michiue $T$, Ishikawa $T$, Quan $L$, et al. Single-stranded DNA as an immunohistochemical marker of neuronal damage in human brain: an analysis of autopsy material with regard to the cause of death. Forensic Sci Int 2008;178:185-191.

18. Naruse I, Keino H, Kawarada Y. Antibody against single-stranded DNA detects both programmed cell death and drug-induced apoptosis. Histochemistry 1994;101:73-78.

19. Umemura S, Osamura RY, Tsutsumi Y. 'Magentosis' in human lactating breast: a mode of cell death accumulating single-stranded DNA stretches or breaks. Pathol Int 1996;46:122-129.

20. Inoue $\mathrm{K}$, Takano $\mathrm{H}$, Yanagisawa $\mathrm{R}$, et al. Protective role of interleukin-6 in coagulatory and hemostatic disturbance induced by lipopolysaccharide in mice. Thromb Haemost 2004:91:1194-1201.

21. Inoue $\mathrm{K}$, Takano $\mathrm{H}$, Shimada $\mathrm{A}$, et al. Role of metallothionein in coagulatory disturbance and systemic inflammation induced by lipopolysaccharide in mice. FASEB J 2006;20:533-535.

22. Weiss SJ. Tissue destruction by neutrophils. N Engl J Med 1989;320:365-376.

23. Chapman HA, Riese RJ, Shi GP. Emerging roles for cysteine proteases in human biology. Annu Rev Physiol 1997;59:63-88.

24. Deng $X$, Wang $X$, Lasson $A$, et al. The involvement of multiple proteaseantiprotease systems and gut origin sepsis in zymosan-associated endothelial barrier injury and multiple organ dysfunction in rats. Shock 2001;16:298-303.

25. Leonis MA, Toney-Earley K, Degen SJ, et al. Deletion of the Ron receptor tyrosine kinase domain in mice provides protection from endotoxin-induced acute liver failure. Hepatology 2002;36: 1053-1060.

26. Lin SD, Endo R, Sato A, et al. Plasma and urine levels of urinary trypsin inhibitor in patients with acute and fulminant hepatitis. J Gastroenterol Hepatol 2002;17:140-147.

27. Wong F, Bernardi M, Balk R, et al. Sepsis in cirrhosis: report on the 7th meeting of the International Ascites Club. Gut 2005;54:718-725.

28. Aosasa S, Ono S, Seki S, et al. Inhibitory effect of protease inhibitor on endothelial cell activation. J Surg Res 1998;80:182-187.

29. Eipel C, Bordel R, Nickels RM, et al. Impact of leukocytes and platelets in mediating hepatocyte apoptosis in a rat model of systemic endotoxemia. Am J Physiol Gastrointest Liver Physiol 2004;286: G769-G776.

30. Jaeschke H, Fisher MA, Lawson JA, et al. Activation of caspase 3 (CPP32)-like proteases is essential for TNF-alpha-induced hepatic parenchymal cell apoptosis and neutrophil-mediated necrosis in a murine endotoxin shock model. J Immunol 1998;160:3480-3486.

31. Liu D, Li C, Chen Y, et al. Nuclear import of proinflammatory transcription factors is required for massive liver apoptosis induced by bacterial lipopolysaccharide. J Biol Chem 2004;279:48434-48442.

32. Hemmer CJ, Lehr HA, Westphal K, et al. Plasmodium falciparum Malaria: reduction of endothelial cell apoptosis in vitro. Infect Immun 2005;73:1764-1770.

33. Koizumi R, Kanai $\mathrm{H}$, Maezawa $\mathrm{A}$, et al. Therapeutic effects of ulinastatin on experimental crescentic glomerulonephritis in rats. Nephron 2000;84:347-353. 